\title{
Effect of Vacuum Energy on Evolution of Primordial Black Holes in Einstein Gravity
}

\author{
Bibekananda Nayak ${ }^{a *}$ and Mubasher Jamil ${ }^{b \dagger}$ \\ ${ }^{a}$ Department of Physics, Utkal University, Vanivihar, Bhubaneswar 751004, India \\ ${ }^{b}$ Center for Advanced Mathematics and Physics, National University of Sciences and Technology, H-12, Islamabad, Pakistan \\ Email : *bibeka@iopb.res.in and ${ }^{\dagger}$ mjamil@camp.nust.edu.pk
}

\begin{abstract}
We study the evolution of primordail black holes by considering present universe is no more matter dominated rather vacuum energy dominated. We also consider the accretion of radiation, matter and vacuum energy during respective dominance period. In this scenario, we found that radiation accretion efficiency should be less than 0.366 and accretion rate is much larger than previous analysis by B. Nayak et al. [1]. Thus here primordial black holes live longer than previous works [1]. Again matter accretion slightly increases the mass and lifetime of primordial black holes. However, the vacuum energy accretion is slightly complicated one, where accretion is possible only upto a critical time. This critical time depends on the values of accretion efficiency and formation time. If a primordial black hole lives beyond critical time, then its' lifespan increases due to vacuum energy accretion. But for presently evaporating primordial black holes, critical time comes much later than their evaporating time and thus vacuum energy could not affect those primordial black holes. We again found that the constraints on the initial mass fraction of PBH obtained from the $\gamma$-ray background limit becomes stronger in the presence of vacuum energy.
\end{abstract}

PACS numbers: 04.20.Fy; 04.50.+h; 98.80.-k

Keywords: primordial black holes, vacuum energy, accretion

\section{INTRODUCTION}

Black holes which are formed in the early universe are known as Primordial Black Holes (PBHs). A comparison of the cosmological density of the universe at any time after the Big Bang with the density associated with a black hole shows that PBHs would have of order the particle horizon mass. PBHs could thus span enormous mass range starting from $10^{-5} \mathrm{gm}$ to more than $10^{15} \mathrm{gm}$. These black holes are formed as a result of initial inhomogeneities [2, 3], inflation [4, 5], phase transitions [6], bubble collisions [7, 8], or the decay of cosmic loops [9]. In 1974 Hawking discovered that the black holes emit thermal radiation due to quantum effects [10]. So the black holes get evaporated depending upon their masses. But in references [1], it is shown that evaporation of primordial black holes delayed due to accretion of radiation by assuming standard picture of Cosmology. Similar kind of works have been done by many other authors [1] 13].

In standard picture of Cosmology [14], universe is radiation dominated in early period of evolution and is matter dominated now. So universe is undergoing a decelerated expansion through out its evolution. But observations of distant supernovae and cosmic microwave background anisotropy indicates that the present universe is undergoing accelerating expansion [15]. To explain this unwanted observational fact it is thought that present universe is dominated by vacuum energy with negative pressure termed as dark energy. SN Ia observations also provide the evidence of a decelerated universe in the recent past with trasition from decelerated to accelerated occuring at redshift $z_{q=0} \sim 0.5$ [16]. So the vacuum energy domination should be started from $z_{q=0} \sim 0.5$ i.e. $t_{q=0} \sim \frac{1}{2} t_{0}$.

In this work, we study the evolution of $\mathrm{PBH}$ by considering present universe is vacuum energy dominated. Here we consider accretion of radiation, matter and vacuum energy during respective dominance period and mainly discuss how accretion of vacuum energy affect PBH evolution.

\section{PRIMORDIAL BLACK HOLES AND EINSTEIN'S GRAVITY}

For a spatially flat FRW Universe with scale factor $a$, the first Friedmann equation is

$$
\left(\frac{\dot{a}}{a}\right)^{2}=\frac{8 \pi G}{3} \rho
$$

and

the total energy conservation equation is

$$
\dot{\rho}+3 H(\rho+p)=0
$$


where $H=\frac{\dot{a}}{a}$ is the Hubble parameter, $\rho$ is the total energy density and $p$ is the total pressure of the background fluid. Here we assume the equation of state $p=w \rho$ for the cosmic fluids i.e. radiation $(w=1 / 3)$, matter $w=0$ and vacuum energy $w=-1$. The Universe evolves from an initial radiation $\left(t<t_{1}\right)$ to matter $\left(t_{1}<t<t_{2}\right)$ and finally to vacuum energy phase $\left(t>t_{2}\right)$.

Now equation (2) gives [14, 17]

$$
\rho(a) \propto \begin{cases}a^{-4} & \left(t<t_{1}\right), \\ a^{-3} & \left(t_{1}<t<t_{2}\right), \\ c_{1} & \left(t>t_{2}\right) .\end{cases}
$$

Using (3) in (1), we obtain [14, 17]

$$
a(t) \propto \begin{cases}t^{1 / 2} & \left(t<t_{1}\right), \\ t^{2 / 3} & \left(t_{1}<t<t_{2}\right), \\ e^{H_{0} t} & \left(t>t_{2}\right), \text { where } H_{0}=\sqrt{\frac{8 \pi G \rho_{c}}{3}} .\end{cases}
$$

Due to Hawking radiation, the rate at which the $\mathrm{PBH}$ mass decreases is given by

$$
\dot{M}_{\text {evap }}=-\frac{a_{H}}{256 \pi^{3}} \frac{1}{G^{2} M^{2}},
$$

where $a_{H}$ is the black body constant.

Again PBH mass can be changed by accumulating radiation, matter or vacuum energy at a rate given by

$$
\dot{M}(t)_{\text {acc }}=16 \pi G^{2} f_{i} M^{2} \rho_{i},
$$

where $f$ is the accretion efficiency and $\rho$ is the density. Subscript $i$ indicates radition, matter or vacuum energy.

\section{DIFFERENT ACCRETION REGIMES}

\section{A. Accretion of radiation}

When a $\mathrm{PBH}$ accretes radiation, the equation governing this accretion is

$$
\dot{M}(t)_{\mathrm{acc}}=16 \pi G^{2} f_{\text {rad }} M^{2} \rho_{r} .
$$

Making use of equation (3) i.e. $\rho_{r}=\rho_{r}^{0}\left(\frac{a}{a_{0}}\right)^{-4}$ and equation (4), we get

$$
\dot{M}(t)_{\mathrm{acc}}=16 \pi G^{2} f_{\text {rad }} \rho_{\mathrm{cr}} \Omega_{r}^{0} M^{2}\left(\frac{t}{t_{1}}\right)^{-2}\left(\frac{t_{1}}{t_{2}}\right)^{-8 / 3} e^{-4 H_{0}\left(t_{2}-t_{0}\right)} .
$$

On integration, equation (8) gives

$$
M(t)=M_{i}\left[1+16 \pi G^{2} f_{r a d} \rho_{\mathrm{cr}} \Omega_{r}^{0} M_{i}\left(\frac{1}{t_{1}}\right)^{-2}\left(\frac{t_{1}}{t_{2}}\right)^{-8 / 3} e^{-4 H_{0}\left(t_{2}-t_{0}\right)}\left(\frac{1}{t}-\frac{1}{t_{i}}\right)\right]^{-1},
$$

where $M_{i}$ is an initial mass of PBH at time $t_{i}$. The superscript 0 refers to present value of physical quantities. Eq. (9) determines the evolution of $\mathrm{PBH}$ by the accretion of radiation.

Using horizon mass, which varies with time as $M_{H}(t)=G^{-1} t$, as initial mass of $\mathrm{PBH}$ and inserting numerical values of different quantities like $G=6.67 \times 10^{-8}$ dyne- $\mathrm{cm}^{2} / \mathrm{gm}^{2}, \rho_{c}=1.1 \times 10^{-29} \mathrm{gm} / \mathrm{cm}^{3}, t_{1}=10^{11} s, t_{2}=0.5 \times t_{0}$ with $t_{0}=4.42 \times 10^{17} s$ and $\Omega_{r}^{0}=10^{-5}$, we get

$$
M(t)=M_{i}\left[1+2.729 f_{\text {rad }}\left(\frac{t_{i}}{t}-1\right)\right]^{-1} .
$$

For large time $t$, this equation asymptotes to

$$
M(t)=M_{i}\left[1-2.729 f_{\text {rad }}\right]^{-1}
$$

which gives for accretion to be effective $f<\frac{1}{2.729} \approx 0.366$.

The variation of accreting mass with time is shown in figure- 1 for different accretion efficiencies. The figure shows that the mass of the PBH increases with increase in accretion efficiency. For a particular accretion efficiency, the mass of the PBH increases for a small period of time and then it becomes constant. 


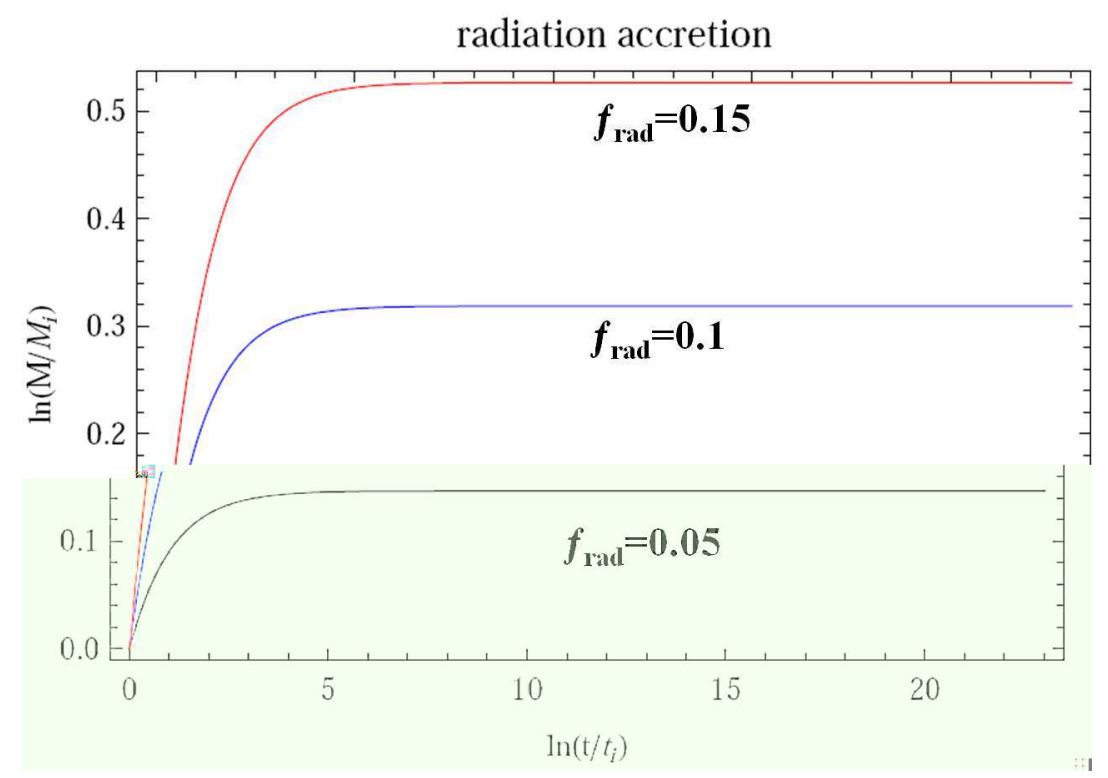

FIG. 1: Variation of PBH mass for $f_{\text {rad }}=0.05,0.1,0.15$

\section{B. Accretion of matter}

In matter dominated era, $\mathrm{PBH}$ mass can increase by absorbing sorrounding matter at a rate

$$
\dot{M}(t)_{\text {acc }}=16 \pi G^{2} f_{m a t} M^{2} \rho_{m} .
$$

In terms of dimensionless density parameters equation (12) can be written by using equations (3) and equation (44) as

$$
\dot{M}(t)_{\mathrm{acc}}=16 \pi G^{2} f_{m a t} M^{2} \rho_{\mathrm{cr}} \Omega_{m}^{0}\left(\frac{t}{t_{2}}\right)^{-2} e^{-3 H_{0}\left(t_{2}-t_{0}\right)} .
$$

Integrating equation (13), we get

$$
M(t)=M_{i}\left[1+16 \pi G^{2} f_{m a t} \rho_{\mathrm{cr}} t_{2}^{2} M_{i} \Omega_{m}^{0} e^{-3 H_{0}\left(t_{2}-t_{0}\right)}\left(\frac{1}{t}-\frac{1}{t_{i}}\right)\right]^{-1} .
$$

Taking horizon mass as initial mass of $\mathrm{PBH}$ and using different numerical values along with $\Omega_{m}^{0}=0.04$, one can find

$$
M(t)=M_{i}\left[1+0.372 f_{\text {mat }}\left(\frac{1}{t}-\frac{1}{t_{i}}\right)\right]^{-1} .
$$

For large time $t$, this equation gives

$$
M(t)=M_{i}\left[1-0.372 f_{m a t}\right]^{-1} .
$$

which gives $f_{\text {mat }}$ can take any value between 0 and 1 .

The variation of accreting mass for different accretion efficiencies is shown in figure-2. This figure indicates that the mass of the $\mathrm{PBH}$ varies in a similar fashion as radiation accretion, but here variation is very small.

\section{Accretion of vacuum energy}

The presence of vacuum energy affected the mass of the PBH at a rate given by

$$
\dot{M}(t)_{\mathrm{acc}}=16 \pi G^{2} f_{\text {vac }} M^{2} \rho_{\Lambda} .
$$

Using equations (3) and (4), we get

$$
\dot{M}(t)_{\mathrm{acc}}=16 \pi G^{2} f_{v a c} M^{2} \rho_{c r} \Omega_{\Lambda} .
$$




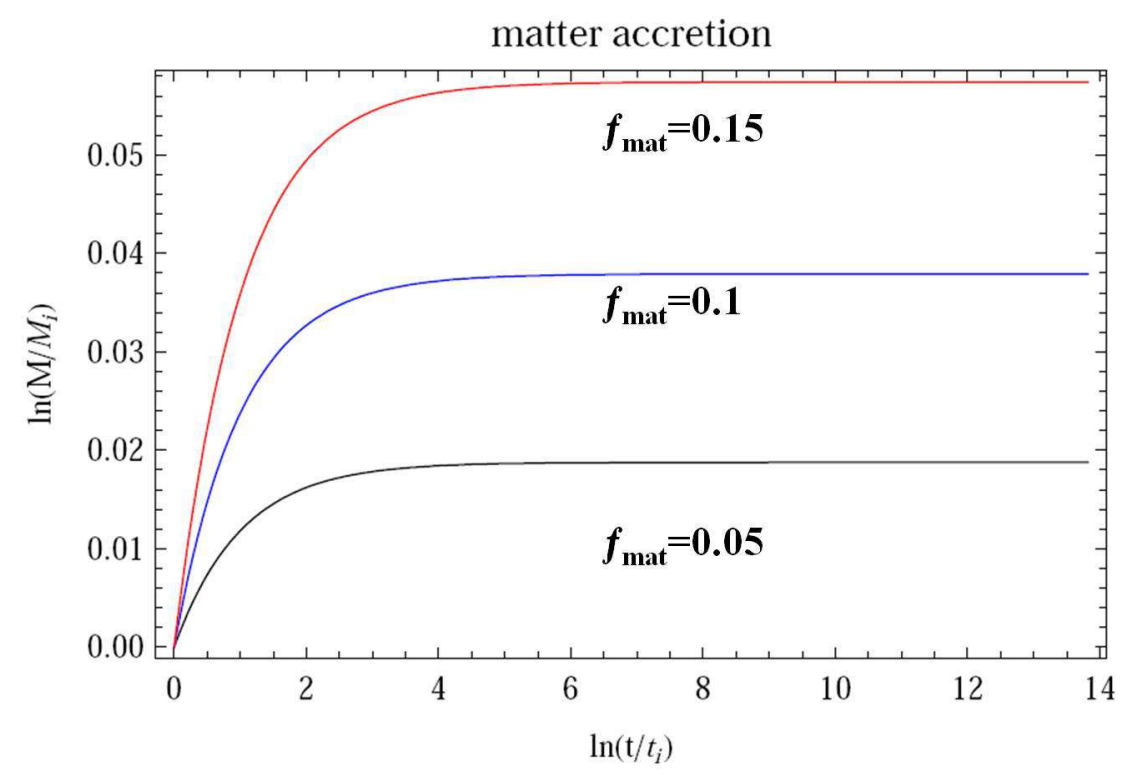

FIG. 2: Variation of PBH mass for $f_{m a t}=0.05,0.1,0.15$

By integrating equation (18), one can find

$$
M(t)=M_{i}\left[1+16 \pi G^{2} f_{v a c} M_{i} \rho_{c r} \Omega_{\Lambda}\left(t_{i}-t\right)\right]^{-1} .
$$

Using numerical values of different quantities along with $\Omega_{\Lambda}=0.73$, we get

$$
M(t)=M_{i}\left[1-2.69 \times 10^{-73} f_{v a c} M_{i}\left(t-t_{i}\right)\right]^{-1} .
$$

For validity of the above equation, time should be less than a critical value $\left(t_{c}\right)$ such that

$$
2.69 \times 10^{-73} f_{\text {vac }} M_{i}\left(t_{c}-t_{i}\right)<1,
$$

which gives

$$
t_{c}<\frac{3.717 \times 10^{72}}{f_{v a c} M_{i}}+t_{i} .
$$

But if a PBH formed before vacuum dominated era, then its' accreting mass becomes

$$
M(t)=M\left(t_{2}\right)\left[1-2.69 \times 10^{-73} f_{v a c} M\left(t_{2}\right)\left(t-t_{2}\right)\right]^{-1},
$$

which leads to

$$
t_{c}<\frac{3.717 \times 10^{72}}{f_{\text {vac }} M\left(t_{2}\right)}+t_{2} .
$$

The variation of accreting mass for different accretion efficiencies is shown in figure-3. This figure shows that at a particular time mass of the $\mathrm{PBH}$ increases with increase in accretion efficiency.

\section{PBH EVOLUTION IN DIFFERENT ERA}

\section{A. Radiation dominated era}

In radiation dominated era, the mass of the $\mathrm{PBH}$ varies accordingly equation

$$
\dot{M}(t)_{\mathrm{PBH}}=16 \pi G^{2} f_{\text {rad }} \rho_{\mathrm{cr}} \Omega_{r}^{0} M^{2}\left(\frac{t}{t_{1}}\right)^{-2}\left(\frac{t_{1}}{t_{2}}\right)^{-8 / 3}-\frac{a_{H}}{256 \pi^{3}} \frac{1}{G^{2} M^{2}} .
$$

This equation can not be solved exactly. But we integrate it by using numerical methods. From our result, we construct the Table-1 for a particular PBH evaporated in the radiation dominated era.

From the Table-1, we found that the lifetime of PBHs increase with increase in radiation accretion efficiency. 


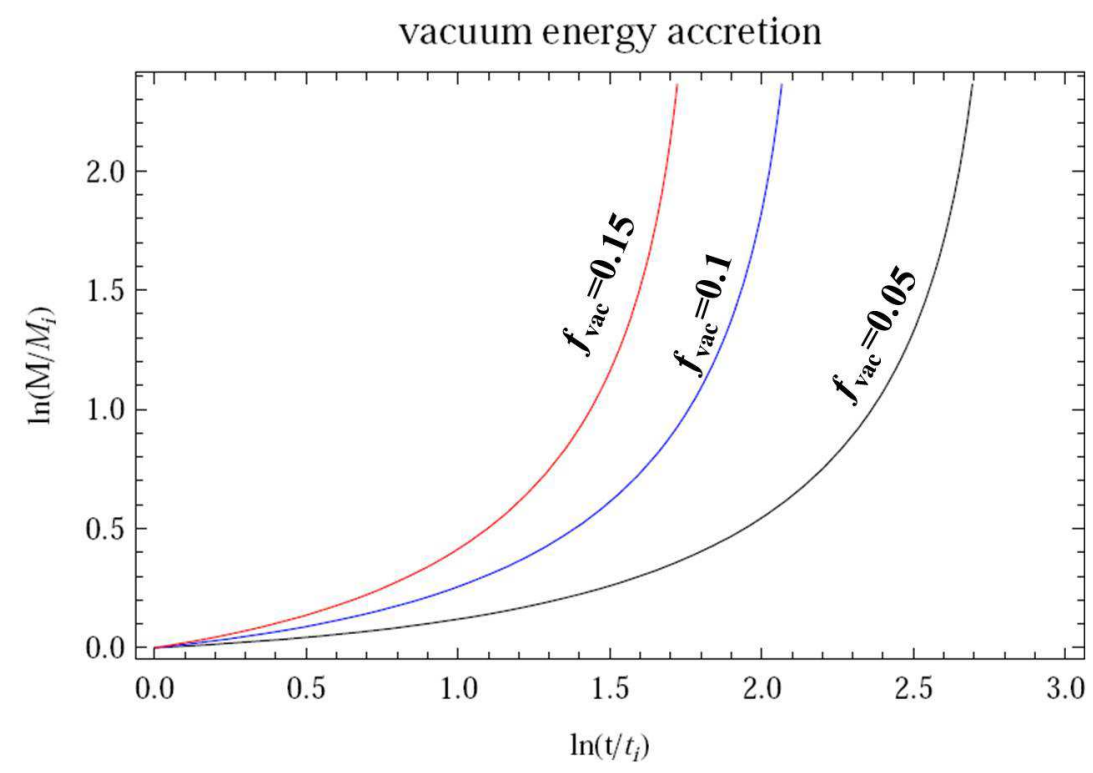

FIG. 3: Variation of PBH mass for $f_{v a c}=0.05,0.1,0.15$

\begin{tabular}{|c|c|}
\hline \multicolumn{2}{|c|}{$t_{i}=10^{-27} s$} \\
\hline$f_{\text {rad }}$ & $t_{\text {evap }}$ \\
\hline 0 & $3.33 \times 10^{4} \mathrm{~s}$ \\
\hline 0.1 & $8.67 \times 10^{4} \mathrm{~s}$ \\
\hline 0.2 & $3.55 \times 10^{5} \mathrm{~s}$ \\
\hline 0.3 & $5.59 \times 10^{6} \mathrm{~s}$ \\
\hline 0.35 & $3.69 \times 10^{8} \mathrm{~s}$ \\
\hline
\end{tabular}

TABLE I: The evaporating times of the PBHs which are created at $t=10^{-27} \mathrm{~s}$ are displayed for several radiation accretion efficiencies.

\section{B. Matter dominated era}

Generally PBHs are not formed in matter dominated era, so here we consider about the PBHs which are formed in radiation dominated era.

When a PBH passes through matter dominated era, it's mass varies as

$$
\dot{M}(t)_{\mathrm{PBH}}=16 \pi G^{2} f_{m a t} M^{2} \rho_{\mathrm{cr}} \Omega_{m}^{0}\left(\frac{t}{t_{2}}\right)^{-2}-\frac{a_{H}}{256 \pi^{3}} \frac{1}{G^{2} M^{2}} .
$$

Solving equation (26) along with equation (25) numerically, we construct the Table-2 for a particular PBH evaporating in matter dominated era.

\begin{tabular}{|c|c|}
\hline \multicolumn{2}{|c|}{$t_{i}=10^{-25} s$ and $f_{\text {rad }}=0.35$} \\
\hline$f_{\text {mat }}$ & $t_{\text {evap }}$ \\
\hline 0 & $3.69 \times 10^{14} \mathrm{~s}$ \\
\hline 0.25 & $3.69 \times 10^{14} \mathrm{~s}$ \\
\hline 0.5 & $3.69 \times 10^{14} \mathrm{~s}$ \\
\hline 0.75 & $3.69 \times 10^{14} \mathrm{~s}$ \\
\hline 1 & $3.69 \times 10^{14} \mathrm{~s}$ \\
\hline
\end{tabular}

TABLE II: The evaporating times of the PBHs which are created at $t=10^{-25} s$ are displayed for several matter accretion efficiencies for a constant radiation accretion.

Table-2 shows that PBH evolution is not much affected by accretion of matter. 


\section{Vacuum energy dominated era}

In this era, the mass of the $\mathrm{PBH}$ varies at a rate given by

$$
\dot{M}(t)_{\mathrm{PBH}}=16 \pi G^{2} f_{v a c} M^{2} \rho_{\mathrm{cr}} \Omega_{\Lambda}^{0}-\frac{a_{H}}{256 \pi^{3}} \frac{1}{G^{2} M^{2}} .
$$

But accretion term is only valid upto a critical time $t_{c}$. After time $t_{c}$ PBH undergoes only evaporation. i.e.

$$
\dot{M}(t)_{\mathrm{PBH}}=-\frac{a_{H}}{256 \pi^{3}} \frac{1}{G^{2} M^{2}} .
$$

Since PBHs are not formed during vacuum energy dominated era, here we only consider the PBH which are formed in radiation dominated era.

Solving equations (24), (25), (26), (27) and (28), we construct the Table-3 for a particular PBH evaporating in vacuum energy dominated era. It is found from the table that accretion of vacuum energy increases the lifespan of PBH. But evaporating time is independent of accretion efficiency. Because with increase in accretion efficiency, critical time $\left(t_{c}\right)$ decreases and $f_{v a c} t_{c}$ remains constant. Again critical time $\left(t_{c}\right)$ for each accretion comes before evaporating time, so complete accretion is possible for all accretion efficiencies.

\begin{tabular}{|c|c|c|}
\hline \multicolumn{3}{|c|}{$t_{i}=10^{-10} s, f_{\text {rad }}=0.35$ and $f_{\text {mat }}=1$} \\
\hline$f_{\text {vac }}$ & $t_{c}$ & $t_{\text {evap }}$ \\
\hline 0 & - & $3.695 \times 10^{59} \mathrm{~s}$ \\
\hline 0.25 & $6.668 \times 10^{43} \mathrm{~s}$ & $1.867 \times 10^{71} \mathrm{~s}$ \\
\hline 0.5 & $3.334 \times 10^{43} \mathrm{~s}$ & $1.867 \times 10^{71} \mathrm{~s}$ \\
\hline 0.75 & $2.223 \times 10^{43} \mathrm{~s}$ & $1.867 \times 10^{71} \mathrm{~s}$ \\
\hline 1 & $1.667 \times 10^{43} \mathrm{~s}$ & $1.867 \times 10^{71} \mathrm{~s}$ \\
\hline
\end{tabular}

TABLE III: The evaporating times of the PBHs which are created at $t=10^{-10} s$ are displayed for several vacuum energy accretion efficiencies for a constant radiation and matter accretion.

\section{CONSTRAINTS ON PBH}

Observed astrophysical constraints arise from the presently evaporating PBHs. So here we discuss about the PBHs whose evaporating time is $t_{0}$. Solving equations (25), (26), (27) and (28) numerically, we construct the Table-4 for presently evaporating PBHs.

\begin{tabular}{|c|c|c|}
\hline \multicolumn{3}{|c|}{$t_{\text {evap }}=t_{0}=4.42 \times 10^{17} s$ and $f_{\text {mat }}=1$} \\
\hline$f_{\text {rad }}$ & $M_{i}$ & $\left(M_{i}\right)_{\text {vac }}$ \\
\hline 0 & $2.367 \times 10^{15} \mathrm{~g}$ & $2.367 \times 10^{15} \mathrm{~g}$ \\
\hline 0.1 & $1.721 \times 10^{15} \mathrm{~g}$ & $1.721 \times 10^{15} \mathrm{~g}$ \\
\hline 0.2 & $1.075 \times 10^{15} \mathrm{~g}$ & $1.075 \times 10^{15} \mathrm{~g}$ \\
\hline 0.3 & $0.429 \times 10^{15} \mathrm{~g}$ & $0.429 \times 10^{15} \mathrm{~g}$ \\
\hline 0.35 & $0.106 \times 10^{15} \mathrm{~g}$ & $0.106 \times 10^{15} \mathrm{~g}$ \\
\hline
\end{tabular}

TABLE IV: The formation masses of the PBHs which are evaporating now are displayed for several accretion efficiencies for both cases without vacuum energy accretion $\left(M_{i}\right)$ and with vacuum energy accretion $\left(M_{i}\right)_{v a c}$.

It is clear from the Table-4 that vacuum energy accretion does not affect lifetimes of presently evaporating PBHs. Case is same for all other PBHs which are completely evaporated by present time.

Now we calculate the constraint which arises from the present $\gamma$-ray background as follows.

The fraction of the Universes' mass going into PBHs at time $t$ is [3]

$$
\beta(t)=\left[\frac{\Omega_{P B H}(t)}{\Omega_{R}}\right](1+z)^{-1},
$$


where $\Omega_{P B H}(t)$ is the density parameter associated with PBHs formed at time $t, z$ is the redshift associated with time $t . \Omega_{R}$ is the microwave background density having value $10^{-4}$.

For $t<t_{1}$, redshift defination implies, $(1+z)^{-1}=\left(\frac{t}{t_{1}}\right)^{\frac{1}{2}}\left(\frac{t_{1}}{t_{2}}\right)^{\frac{2}{3}} e^{H_{0}\left(t_{2}-t_{0}\right)}$.

Now using $M=G^{-1} t$, we can write the fraction of the Universe going into PBHs' as a function of mass M as

$$
\beta(M)=\left(\frac{M}{M_{1}}\right)^{\frac{1}{2}}\left(\frac{t_{1}}{t_{2}}\right)^{\frac{2}{3}} e^{H_{0}\left(t_{2}-t_{0}\right)} \Omega_{P B H}(M) \times 10^{4} .
$$

Observations of the cosmolgical deceleration parameter imply $\Omega_{P B H}(M)<1$ over all mass ranges for which PBHs have not evaporated yet. But presently evaporating $\operatorname{PBHs}\left(M_{*}\right)$ generate a $\gamma$-ray background whose most of the energy is appearing at around $100 \mathrm{Mev}$ [18]. If the fraction of the emitted energy which goes into photons is $\epsilon_{\gamma}$, then the density of the radiation at this energy is expected to be $\Omega_{\gamma}=\epsilon_{\gamma} \Omega_{P B H}\left(M_{*}\right)$. Since $\epsilon_{\gamma} \sim 0.1$ and the observed $\gamma$-ray background density around $100 \mathrm{Mev}$ is $\Omega_{\gamma} \sim 10^{-9}$, one gets $\Omega_{P B H}<10^{-8}$.

Now equation (30), therefore, becomes

$$
\beta\left(M_{*}\right)<\left(\frac{M_{*}}{M_{1}}\right)^{\frac{1}{2}} \times\left(\frac{t_{1}}{t_{2}}\right)^{\frac{2}{3}} e^{H_{0}\left(t_{2}-t_{0}\right)} \times 10^{-4} .
$$

The variation of $\beta\left(M_{*}\right)$ with $f$ drawn from variation of $M_{*}$ with $f$ is shown in the Table- 2 . The bound on $\beta\left(M_{*}\right)$ is strengthened as $f$ approaches its maximum value.

\begin{tabular}{|c|c|c|}
\hline \multicolumn{3}{|c|}{$t_{\text {evap }}=t_{0}$} \\
\hline$f_{\text {rad }}$ & $M_{*}$ & $\beta\left(M_{*}\right)<$ \\
\hline 0 & $2.367 \times 10^{15} \mathrm{~g}$ & $5.23 \times 10^{-26}$ \\
\hline 0.1 & $1.721 \times 10^{15} \mathrm{~g}$ & $4.46 \times 10^{-26}$ \\
\hline 0.2 & $1.075 \times 10^{15} \mathrm{~g}$ & $3.525 \times 10^{-26}$ \\
\hline 0.3 & $0.429 \times 10^{15} \mathrm{~g}$ & $2.227 \times 10^{-26}$ \\
\hline 0.35 & $0.106 \times 10^{15} \mathrm{~g}$ & $1.107 \times 10^{-26}$ \\
\hline
\end{tabular}

TABLE V: Upper bounds on the initial mass fraction of PBHs that are evaporating today for various accretion efficiencies $f$.

But neglecting the presence of vacuum energy, one can find [1]

$$
\beta_{0}\left(M_{*}\right)<\left(\frac{M_{*}}{M_{1}}\right)^{\frac{1}{2}} \times\left(\frac{t_{1}}{t_{0}}\right)^{\frac{2}{3}} \times 10^{-4} .
$$

Comparison of equations (31) and (32) gives

$$
\beta\left(M_{*}\right) \approx 0.364 \times \beta_{0}\left(M_{*}\right) .
$$

Thus the constraint on the initial mass fraction of $\mathrm{PBH}$ obtained from the $\gamma$-ray background limit becomes stronger in the presence of vacuum energy.

\section{CONCLUSION}

Here we study the evolution of primordail black holes by considering present universe is vacuum energy dominated. In our consideration, we have taken that the universe evolves from an initial radiation dominated to matter dominated and finally to present vacuum energy phase. We also consider that when a PBH passes through radiation domination, matter domination and vacuum domination, it accretes radiation, matter and vacuum energy respectively. During radiation dominated era, we found that radiation accretion efficiency should less than 0.366 and accretion rate is much larger than previous works by B. Nayak et al. 11. Thus primordial black holes live longer than previous analysis [1]. In matter dominated era, accretion of matter slightly increases the mass and lifetime of primordial black holes. However, during vacuum energy dominated era, the accumulation of vacuum energy is possible only upto a critical time $t_{c}$. The value of $t_{c}$ depends on accretion efficiency and formation time. If a PBH lives beyond this critical time, then its' lifespan increases due to accretion of vacuum energy. But for presently evaporating PBHs, the critial time $t_{c}$ comes much later than their evaporating time. So those PBHs are not affected by the presence of vacuum energy. We also found that the constraint on the initial mass fraction of PBH obtained from the $\gamma-$ ray background limit becomes stronger in the presence of vacuum energy. 


\section{Acknowledgements}

We are thankful to Prof. L. P. Singh, Utkal University, Bhubaneswar, India for his suggestions which greatly help in preparing this manuscript. B. Nayak would like to thank the Council of Scientific and Industrial Research, Government of India, for the award of SRF, F.No. 09/173(0125)/2007-EMR-I .

[1] B. Nayak and L.P. Singh, A. S. Majumdar, Phys. Rev. D 80, 023529 (2009); B. Nayak and L. P. Singh, Pramana 76, 173 (2011).

[2] Ya. B. Zeldovich and I. Novikov, Sov. Astron. Astrophys. J. 10, 602 (1967).

[3] B. J. Carr, Astrophys. J. 201, 1 (1975).

[4] M. Y. Kholpov, B. A. Malomed and Ya. B. Zeldovich, Mon. Not. R. Astron. Soc. 215, 575 (1985).

[5] B. J. Carr, J. Gilbert and J. Lidsey, Phys. Rev. D 504853 (1994).

[6] M. Y. Kholpov and A. Polnarev, Phys. Lett. 97B, 383 (1980).

[7] H. Kodma, M. Sasaki and K. Sato, Prog. Theor. Phys. 68, 1079 (1982).

[8] D. La and P. J. Steinhardt, Phys. Rev. Lett. 62, 376 (1989).

[9] A. Polnarev and R. Zemboricz, Phys. Rev. D 43, 1106 (1988).

[10] S. W. Hawking, Commun. Math. Phys. 43, 199 (1975).

[11] M. Jamil and U. Debnath, arXiv:1102.2758.

[12] F. C. Adams et. ai., Phys. Lett. B 450, 339 (1999).

[13] J. A. S. Lima, D. C. Guariento and J. E. Horvath, Phys. Lett. B 693, 218 (2010).

[14] S. Wienberg, Gravitation and Cosmology (Wiley, New York, 1972).

[15] A. G. Riess et al., Astron. J. 116, 1009 (1998); S. Perlmutter et al., Astrophys. J. 517, 565 (1999); C. L. Bennett et. al., Astrophys. J. Suppl. Ser. 148, 1 (2003).

[16] M. S. Turner and A. G. Riess, Astrophys. J. 569, 18 (2002): A.G. Riess, Astrophys. J. 560, 49 (2001)

[17] S. Wienberg, Cosmology (Oxford University Press, 2008).

[18] I D Novikov et al., Astron. Astrophys. J. 80, 104 (1979). 